\title{
MODULES OVER PRÜFER DOMAINS
}

\author{
BY \\ SYED M. FAKHRUDDIN
}

\begin{abstract}
We prove that the category of torsion-free modules over a Prüfer domain is equivalent to a certain category of diagrams. As applications we derive (i) a strong version of Baer-Kulikov-Kaplansky theorem for modules over a valuation ring, (ii) the determination of a complete set of invariants for modules of almost countable rank over an almost-maximal valuation ring, (iii) a new nonhomological proof of the characterization of a maximal valuation ring due to Matlis.
\end{abstract}

The following forms part of the author's doctoral dissertation submitted to the Faculty of Graduate Studies, Queen's University, Kingston, Ontario. This was written under the supervision of Professor P. Ribonboim with financial assistance from the Government of Ontario and The Canada Council, to all of whom I express my thanks. I would also like to thank Professor I. Kaplansky for his generous interest in the content of this paper, Dr. I. Fleischer for many stimulating discussions and Dr. W. D. Geyer for helping to improve the presentation.

1. Introduction. Let $R$ be a Prüfer domain with field of quotients $K$. Let $R^{*}$ be a Prüfer domain containing $R$, with field of quotients $K^{*}$, such that $R^{*} \cap K=R$. Clearly $K^{*} \supset K$.

Let $\mathfrak{M}_{R}$ denote the category whose objects are all unitary torsion-free left $R$-modules and whose morphisms are the usual $R$-linear maps; $\mathfrak{M}_{R^{*}}$ is defined similarly. Since all the modules considered in this paper are torsion-free, we will drop that adjective altogether. Cat $(X)$ stands for the usual category of modules over the ring $x$. Although Cat $(X)$ is abelian, $\mathfrak{M}_{R}$ is only additive.

Let $\mathbb{S}$ be the category constructed as follows:

Objects of $\mathfrak{C}$. An object in $\mathbb{C}$ is a diagram of the following type in Cat $(R)$, satisfying the conditions given below.

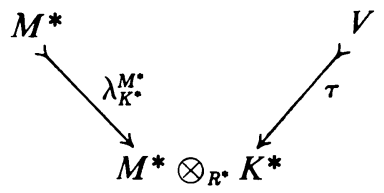

(i) $M^{*} \in \mathfrak{M}_{R^{*}}$;

(ii) $V \in \operatorname{Cat}(K)$ with $\operatorname{Rank}_{R^{*}}\left(M^{*}\right)=\operatorname{dim}_{K}(V)$;

Presented to the Society, November 22, 1969; received by the editors August 6, 1970.

AMS 1970 subject classifications. Primary 13C05.

Key words and phrases. Prüfer domains, categories, equivalence, functor, torsion-free modules, rank, tensor-product, linearly independent sets, completely decomposable.

Copyright (C) 1971, American Mathematical Society 
(iii) $\lambda=\lambda_{K^{*}}^{M^{*}}$ is the canonical $R^{*}$-homomorphism id$M^{*} \otimes \operatorname{inj}_{K^{*}}^{R^{*}}$;

(iv) $\tau$ is a $K$-monomorphism which is rational and ample.

$\tau$ is rational iff every $K$-linearly independent subset of $V$ is mapped into a $K^{*}$ linearly independent subset of $M^{*} \bigotimes_{R^{*}} K^{*}$, and also that $K^{*} \cdot \tau(V)=M^{*} \bigotimes_{R^{*}} K^{*}$. $\tau$ is ample iff

(a) $R^{*} \cdot\left(\tau(V) \cap \lambda\left(M^{*}\right)\right)=\lambda\left(M^{*}\right)$;

(b) $K \cdot\left(\tau(V) \cap \lambda\left(M^{*}\right)\right)=\tau(V)$.

The diagram given above is called a wedge and is denoted by $\mathcal{O} \equiv\left(M^{*}, \tau, V\right)$. $\tau$ is called the twist mapping of $\mathcal{O}$, or simply the twist mapping.

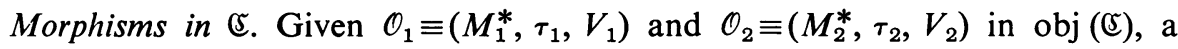
morphism $\phi^{*}$ from $\mathcal{O}_{1}$ to $\mathcal{O}_{2}$ is a triple $\left(\phi_{R^{*}}, \phi_{K^{*}}, \phi_{K}\right)$ where

(i) $\phi_{R^{*}}: M_{1}^{*} \rightarrow M_{2}^{*}$ is $R^{*}$-linear,

(ii) $\phi_{K^{*}}: M_{1}^{*} \otimes_{R^{*}} K^{*} \rightarrow M_{2}^{*} \otimes_{R^{*}} K^{*}$ is $K^{*}$-linear,

(iii) $\phi_{K}: V_{1} \rightarrow V_{2}$ is $K$-linear, such that the following diagram commutes:

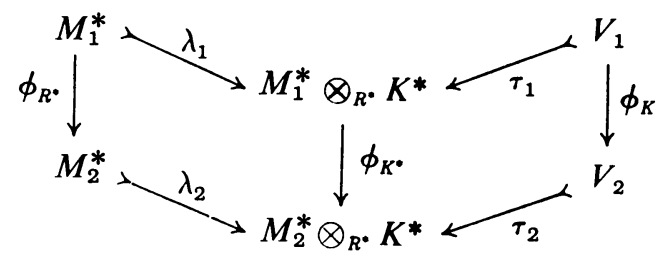

Hence we have

(a) $\lambda_{2} \circ \phi_{R^{*}}=\phi_{K^{*}} \circ \lambda_{1}$ as $R^{*}$-linear maps, and

(b) $\tau_{2} \circ \phi_{K}=\phi_{K^{*}} \circ \tau_{1}$ as $K$-linear maps.

We will call the pair of relations (a) and (b) the "basic relations for a ङ-morphism" or simply the basic relations.

It is easy to check that $\mathbb{E}$ is a category; it is even an additive category. Since Cat $\left(R^{*}\right)$, Cat $\left(K^{*}\right)$, Cat $(K)$ are additive categories, the necessary conditions for the additivity of $\mathbb{C}$ can be checked by componentwise operations on $\mathbb{E}$-morphisms.

In this paper we prove

THEOREM A. $\mathfrak{M}_{R}$ and $\mathfrak{\complement}$ are equivalent categories.

We given many applications of this theorem. To wit: (i) a strong version of the Baer-Kulikov-Kaplansky theorem for modules over valuation rings, (ii) the determination of a complete set of invariants for modules of at most countable rank over an almost-maximal valuation ring, (iii) a new nonhomological proof of the characterization of a maximal valuation ring due to Matlis.

\section{Preliminaries.}

2.1. LEMMA. If $M \in \mathfrak{M}_{R}$ and $M^{*}=M \otimes_{R} R^{*}$ then (a)

$$
0 \rightarrow M \cong M \otimes_{R} R \stackrel{\lambda_{R^{*}}^{M}}{\longrightarrow} M \otimes R^{*}=M^{*}
$$

is exact, where $\lambda_{R^{*}}^{M}=\mathrm{id}_{M} \otimes \operatorname{inj}_{R^{*}}^{R}$, and (b) $R^{*} \cdot \lambda_{R^{*}}^{M}(M)=M^{*}$. 
Proof. Since $M \in \mathfrak{M}_{R}$ and $R$ is a Prüfer domain, $M$ is $R$-flat. Hence (a) follows from the exact sequence $0 \rightarrow R \rightarrow R^{*}$ and (b) is clear.

2.2. Proposition. Let $M \in \mathfrak{M}_{R}$ and $N \in \mathfrak{M}_{R^{*}}$. Suppose there exists an $R$-linear map $\phi: M \rightarrow N$ such that (i) $\phi$ carries every $R$-linearly independent set in $M$ to an $R^{*}$-linearly independent set in $N$ and (ii) $R^{*} \cdot \phi(M)=N$.

Then there exists a unique $R^{*}$-linear map $\phi: M \otimes R^{*} \rightarrow N$ such that $\phi \circ \lambda_{R^{*}}^{M}=\phi$, and $\phi$ is an isomorphism.

Proof. By the universal property of tensor product, there exists a unique $R^{*}$ linear map $\phi$ such that $\phi \circ \lambda_{R^{*}}^{M}=\phi$. By assumption (ii) $\phi$ is clearly surjective.

The map $\phi$ is given explicitly by $\phi\left(\sum x_{i} \otimes r_{i}\right)=\sum r_{i} x_{i} \in N$. Let $\sum x_{i} \otimes r_{i} \in M^{*}$ be such that $\phi\left(\sum x_{i} \otimes r_{i}\right)=0$, and consider $M_{0}=\sum R x_{i} \subseteq M$. If $\phi_{0}: M_{0} \rightarrow N$ is the restriction of $\phi$ to $M_{0}$, then $\phi_{0}: M_{0} \otimes R^{*} \rightarrow N$ is the corresponding restriction of $\phi$, since $R^{*}$ is $R$-flat and so $M_{0} \otimes R^{*} \subseteq M \otimes R^{*}$. Let $K=\operatorname{ker} \phi_{0}$ and $N_{0}=\operatorname{im} \phi_{0}$. Then $\sum x_{i} \otimes r_{i} \in K$ and we have the exact sequence

$$
0 \rightarrow K \rightarrow M_{0}^{*} \rightarrow N_{0} \rightarrow 0 .
$$

By construction, $M_{0}$ is an $R$-module of finite rank, say $n$. Hence $M_{0}^{*}$ has $R^{*}$-rank $n$. (See [2, Lemma 4.3].) Now $\phi_{0}$ satisfies both the hypotheses of the proposition. By hypothesis (i) $\phi_{0}$ does not decrease the rank. Therefore, $\operatorname{Rank}_{R^{*}}\left(R^{*} \cdot \phi_{0}\left(M_{0}\right)\right)$ $=\operatorname{Rank}_{R^{*}}\left(\phi_{0}\left(M_{0}^{*}\right)\right)=\operatorname{Rank}_{R^{*}}\left(N_{0}\right) \geqq n$. By the additivity of ranks and the fact that $K$ is torsion-free, we have $K=0$. Thus $\sum r_{i} \otimes m_{i}=0$, proving that $\phi$ is also injective. Q.E.D.

2.3. Proposition. Consider the following diagram in Cat $(R)$ with the data given below:

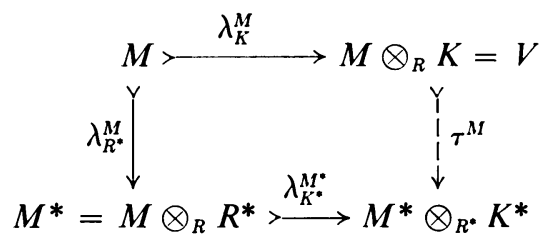

(i) $M \in \mathfrak{M i}_{R}$; (ii) the $\lambda$ 's stand for the corresponding tensor maps with $\mathrm{id}_{M}$ (or $\mathrm{id}_{M}$ * respectively); (iii) $\tau^{M}$ is the unique $K$-linear map induced by the $R$-linear map

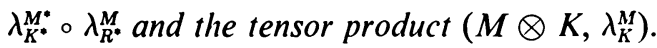

Then the diagram is a pullback in Cat $(R)$.

Proof. Clearly by construction $\tau^{M} \circ \lambda_{K}^{M}=\lambda_{K^{*}}^{M^{*}} \circ \lambda_{R^{*}}^{M}$. Also $\tau^{M}$ is injective, since each $\lambda$ is injective. Hence to prove the proposition, it is enough to prove that

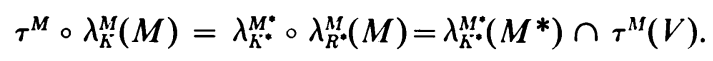

Since $R^{*}+K=K^{*}$ and $R^{*} \cap K=R$, the result follows from [1, Lemma 7, p. 32]. 
2.4. Proposition. Given an object $\mathcal{O} \equiv\left(M^{*}, \tau, V\right)$ in $\mathbb{C}$ (which is a diagram in Cat $((R)$ let $M$ be its pullback in Cat $(R)$.

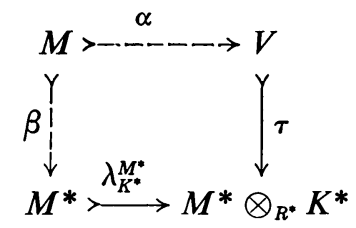

Then the following conditions hold:

(i) $\alpha$ and $\beta$ are injective and $M \in \mathfrak{M}_{R}$.

(ii) $K \alpha(M)=V$.

(iii) $R^{*} \beta(M)=M^{*}$.

(iv) $\beta$ carries each $R$-linearly independent set of elements of $M$ into an $R^{*}$-linearly independent set of $M^{*}$.

Proof. (i) The diagram lies in Cat $(R)$, which is an abelian category. $\tau$ and $\lambda_{K^{*}}^{M^{*}}$ are injective maps, hence $\alpha$ and $\beta$ are injective. Also $\alpha(M)$ is a submodule of a torsion-free module $V$, so $M$ is torsion-free, thus $M \in \mathfrak{M}_{R}$.

(ii) $K \cdot\left(\lambda\left(M^{*}\right) \cap \tau(V)\right)=\tau(V)$ is one of the ampleness conditions on $\mathfrak{乛}$. Now $M$ is the pullback. Hence $\tau \circ \alpha(M)=\left(\lambda\left(M^{*}\right) \cap \tau(V)\right)$. Hence $K \cdot \tau \circ \alpha(M)=\tau(K \cdot \alpha(M))$ $=K\left(\lambda\left(M^{*}\right) \cap \tau(V)\right)=\tau(V)$. But $\tau$ is injective, hence $K \cdot \alpha(M)=V$.

(iii) Using the other ampleness condition, we deduce in a similar way that $R^{*} \cdot \beta(M)=M^{*}$.

(iv) Clearly $\lambda$ carries an $R^{*}$-linearly independent set in $M^{*}$ into a $K^{*}$-linearly independent set in $M^{*} \otimes K^{*}$. By assumption $\tau$ carries a $K$-linearly independent subset of $V$ to a $K^{*}$-linearly independent subset of $M^{*} \otimes K^{*}$. Furthermore, if $\sum k_{i} \alpha\left(x_{i}\right)=0$ for $k_{i} \in K$ and $x_{i} \in M$, then there exist $r_{i} \in R$ such that $\sum r_{i} \alpha\left(x_{i}\right)=0$. But then $\alpha\left(\sum r_{i} x_{i}\right)=0$ and $\alpha$ is injective, so $\sum r_{i} x_{i}=0$. Thus $\alpha$ also preserves independence under the extension of scalars from $R$ to $K$. Now by commutativity, $\beta$ carries any $R$-linearly independent set of $M$ into an $R^{*}$-linearly independent set of $M^{*}$. Q.E.D.

\section{Construction of the functor.}

\subsection{The expanding functor $E$.}

Action on objects. Given $M \in \mathfrak{M}_{R}$, consider the diagram constructed in Proposition 2.3. We will prove that $\mathcal{O} \equiv\left(M \otimes R^{*}, \tau^{M}, M \otimes K\right)$ is indeed an object in $\mathfrak{夭}$.

By [1, p. 34, Proposition 8], $M \otimes R^{*}$ is $R^{*}$-flat. But $R^{*}$ is a Prüfer domain, hence $M \otimes R^{*}$ is $R^{*}$-torsion free, and $\operatorname{Rank}_{R^{*}}\left(M \otimes R^{*}\right)=\operatorname{Rank}_{R}(M)=\operatorname{dim}_{K}(M \otimes K)$. (See [2, Lemma 4.3].)

$\tau$ is clearly a monomorphism, as was noted in Proposition 2.3. Now the $\lambda$ 's preserve the linear independence of elements under the extension of the appropriate scalar domains. It follows that $\tau$ carries $K$-linearly independent sets into $K^{*}$ linearly independent sets (cf. Proposition 2.4(iv) above). Thus the rationality of $\tau$ is proved. 
By Proposition 2.3, $M$ is a pullback. Hence we have $\lambda_{K}^{M} \circ \tau^{M}(M)=\lambda_{K^{*}}^{M^{*}} \circ \lambda_{R^{*}}^{M}(M)$ $=\left(\lambda_{K^{*}}^{M^{*}}\left(M \otimes R^{*}\right) \cap \tau^{M}(M \otimes K)\right)$. Now $\lambda_{K^{*}}^{M^{*}}$ is injective and $R^{*} \cdot \lambda_{R^{*}}^{M}(M)=M \otimes R^{*}$, so we have $R^{*} \cdot\left(\lambda_{K^{*}}^{M^{*}}\left(M \otimes R^{*}\right) \cap \tau^{M}(M \otimes K)\right)=\lambda_{K^{*}}^{M^{*}}\left(M \otimes R^{*}\right)$. In a similar way it follows that $K\left(\lambda_{K^{*}}^{M^{*}}\left(M \otimes R^{*}\right) \cap \tau^{M}(M \otimes K)\right)=\tau^{M}(M \otimes K)$. Both the ampleness conditions are therefore true and we have shown that $\mathcal{O} \equiv\left(M \otimes R^{*}, \tau^{M}, M \otimes K\right)$ $\in \mathfrak{C}$. We define $E(M)=\mathcal{O}$.

Action on morphisms. Let $\phi \in \operatorname{Morph}(M, N)$ where $M, N \in \mathfrak{M}_{R}$. Consider the following diagram:

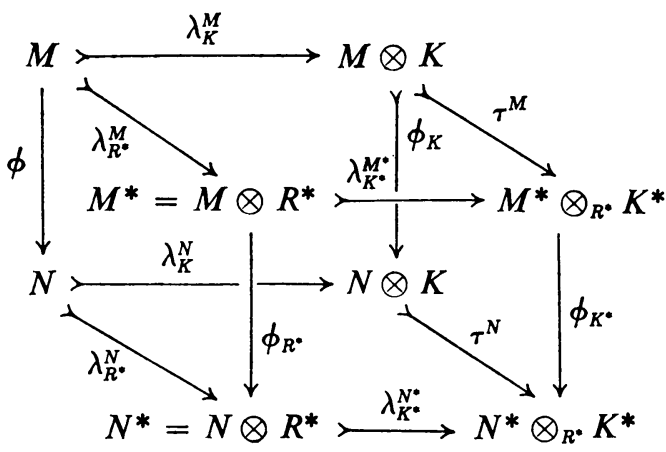

By the universal property of tensor product, we know

(i) there exists a unique $R^{*}$-linear map $\phi_{R^{*}}: M^{*} \rightarrow N^{*}$ such that

$$
\phi_{R^{*}} \circ \lambda_{R^{*}}^{M}=\lambda_{R^{*}}^{N} \circ \phi,
$$

(ii) there exists a unique $K$-linear map $\phi_{K}: M \otimes_{R} K \rightarrow N \otimes K$ such that

$$
\phi_{K} \circ \lambda_{K}^{M}=\lambda_{K}^{N} \circ \phi \text {, }
$$

(iii) there exists a unique $K^{*}$-linear map $\phi_{K^{*}}: M^{*} \otimes_{R^{*}} K^{*} \rightarrow N^{*} \bigotimes_{R^{*}} K^{*}$ such that

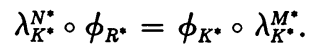

Let $\hat{\phi} \equiv\left(\phi_{R^{*}}, \phi_{K^{*}}, \phi_{K}\right)$. We will show that $\hat{\phi}$ satisfies the basic requirements of a morphism in $\mathfrak{E}$. We have only to show that $\phi_{K^{*}} \circ \lambda_{K^{*}}^{M^{*}}=\lambda_{K^{*}}^{N^{*}} \circ \phi_{R^{*}}$ and $\phi_{K^{*}} \circ \tau^{M}$ $=\tau^{N} \circ \phi_{K}$. The first of these relations is just (iii) above. To prove the second, note that

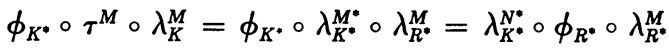

$$
\begin{aligned}
& =\lambda_{K^{*}}^{N^{*}} \circ \lambda_{R^{*}}^{N} \circ \phi=\tau^{N} \circ \lambda_{K}^{N} \circ \phi=\tau^{N} \circ \phi_{K} \circ \lambda_{K}^{M} \text {. }
\end{aligned}
$$

Since $\lambda_{K}^{M}(M)$ generates the $K$-space $M \otimes_{R} K$, we have $\phi_{K^{*}} \circ \tau^{M}=\tau^{N} \circ \phi_{K}$. Now we put $E(\phi)=\hat{\phi}$, thereby defining the action of $E$ on $R$-morphisms in $\mathfrak{M}_{R}$.

It is clear that $E\left(1_{M}\right)=1_{E(M)}$ and $E(\phi \circ \psi)=E(\phi) \circ E(\psi)$. Thus $E$ is a covariant functor. It is equally straightforward to check that $E$ is additive, by using the uniqueness of the component maps in a $\mathbb{E}$-morphism together with the basic relations for $\mathfrak{c}$-morphisms. 
4. Proof of the main theorem. We will now prove that $E$ is an equivalence from $\mathfrak{M R}_{R}$ to $\mathfrak{~}$.

4.1. $E$ is faithful. Since $E$ is additive, it is enough to show that $\phi \in \operatorname{Morph}\left(\mathfrak{M}_{R}\right)$ and $\phi \neq 0$ implies $E(\phi) \neq(0,0,0)$ in $\mathbb{C}$.

Let $0 \neq x \in M$ be such that $\phi(x) \neq 0$ (see the previous diagram). Then $\phi_{R^{*}} \circ \lambda_{R^{*}}^{M}(x)$ $=\lambda_{R^{*}}^{N} \circ \phi(x) \neq 0$, because $\lambda_{R^{*}}^{N}$ is injective, so $\phi_{R^{*}} \neq 0$. By similar arguments, it follows that $\phi_{K} \neq 0$ and $\phi_{K^{*}} \neq 0$. This shows $E(\phi) \neq(0,0,0)$.

4.2. $E$ is full. Consider the following diagram in Cat $(R)$ :

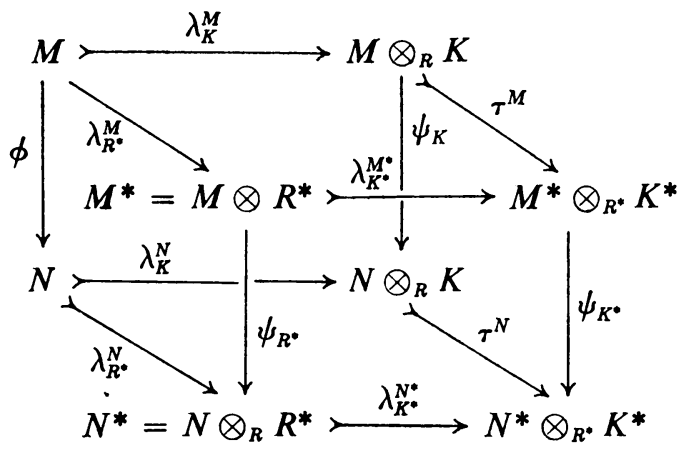

Given a morphism $\left(\psi_{R^{*}}, \psi_{K^{*}}, \psi_{K}\right)$ from $E(M)$ to $E(N)$, we will find $\phi: M \rightarrow N$ such that $E(\phi)=\hat{\phi}=\left(\psi_{R^{*}}, \psi_{K^{*}}, \psi_{K}\right)$.

Define $\alpha=\psi_{K} \circ \lambda_{K}^{M}: M \rightarrow N \otimes K$, and $\beta=\psi_{R^{*}} \circ \lambda_{R^{*}}^{M}: M \rightarrow N \otimes R^{*}$; these are both $R$-linear maps. We also have $\tau^{N} \circ \alpha=\lambda_{K^{*}}^{N^{*}} \circ \beta$ by the commutativity of the diagram.

By Proposition 2.3, the bottom square is a pullback and hence there exists a unique $R$-linear map $\phi: M \rightarrow N$ such that $\lambda_{K}^{N} \circ \phi=\psi_{K} \circ \lambda_{K}^{M}=\alpha$ and $\lambda_{R^{*}}^{N} \circ \phi$ $=\psi_{R^{*}} \circ \lambda_{R^{*}}^{M}=\beta$. If $E(\phi) \equiv\left(\phi_{R^{*}}, \phi_{K^{*}}, \phi_{K}\right)$, then by the uniqueness of the components in a $\left(5\right.$-morphism, we conclude that $\phi_{R^{*}}=\psi_{R^{*}}, \phi_{K^{*}}=\psi_{K^{*}}$ and $\phi_{K}=\psi_{K^{*}}$. In other words, $E(\phi) \equiv\left(\psi_{R^{*}}, \psi_{K^{*}}, \psi_{K}\right)$.

4.3. $E$ is representative. Given an object $\mathcal{O} \equiv\left(M^{*}, \tau, V\right)$ in $\mathfrak{E}$, let $M$ be the pullback of the diagram corresponding to $\mathcal{O}$ in Cat $(R)$. By Proposition 2.4(i) $M \in \mathfrak{M}_{R}$ and we can therefore form $E(M)$. Now consider the following diagram:

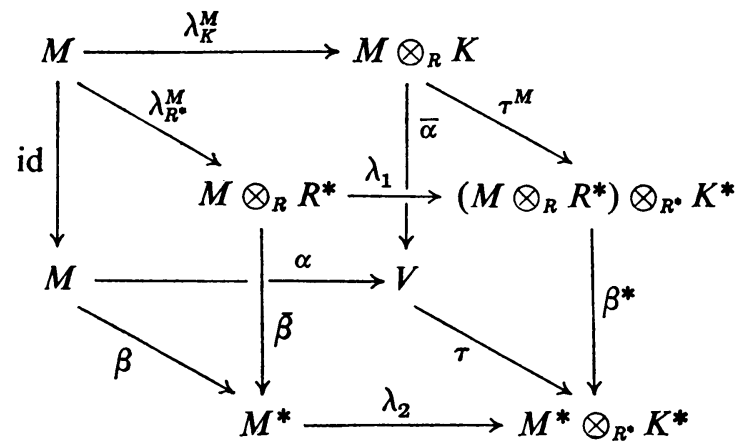


Making use of the three tensor products in the top square of the diagram we have

(i) there exists a unique $R^{*}$-linear map $\bar{\beta}: M \otimes R^{*} \rightarrow M^{*}$ such that

$$
\bar{\beta} \circ \lambda_{R^{*}}^{M}=\beta \circ \mathrm{id}_{M}=\beta,
$$

(ii) there exists a unique $K$-linear map $\bar{\alpha}: M \otimes K \rightarrow V$ such that

$$
\bar{\alpha} \circ \lambda_{K}^{M}=\alpha \circ \mathrm{id}_{M}=\alpha,
$$

(iii) there exists a unique $K^{*}$-linear map $\beta^{*}:\left(M \otimes_{R} R^{*}\right) \otimes_{R^{*}} K^{*} \rightarrow M^{*} \otimes_{R^{*}} K^{*}$ such that

$$
\lambda_{2} \circ \bar{\beta}=\beta^{*} \circ \lambda_{1}
$$

(a) By Proposition 2.4(iii) and (iv), $\beta$ satisfies all the hypotheses of Proposition 2.2. Hence the induced map $\bar{\beta}$ is an isomorphism.

(b) By Proposition 2.4(i), $\alpha$ is injective. Therefore, $\bar{\alpha}$ is also injective. But by 2.4(ii), $K \cdot \alpha(M)=V$, so $\bar{\alpha}$ is surjective as well. Hence $\bar{\alpha}$ is an isomorphism.

(c) $\beta^{*} \circ \tau^{M} \circ \lambda_{K}^{M}=\tau \circ \bar{\alpha} \circ \lambda_{K}^{M}$ by relations (i), (ii), (iii) above and other commutative relations on the diagram. But $\lambda_{K}^{M}(M)$ generates $M \otimes K$, so

$$
\beta^{*} \circ \tau^{M}=\tau \circ \bar{\alpha} .
$$

(d) $\beta^{*}\left(\left(M \otimes_{R} R^{*}\right) \otimes_{R^{*}} K^{*}\right)=\beta^{*}\left(K^{*} \cdot \tau^{M}(M \otimes K)\right)=K^{*}\left(\beta^{*} \circ \tau^{M}(M \otimes K)\right)$

$$
=K^{*} \cdot \tau \circ \bar{\alpha}(M \otimes K)=K^{*} \cdot \tau(V)=M^{*} \bigotimes_{R^{*}} K^{*} .
$$

Thus $\beta^{*}$ is surjective. But $\beta^{*}$ is also injective, since it is the localization of the injective map $\bar{\beta}$. Therefore $\beta^{*}$ is an isomorphism.

The relations (3) and (4) above imply $\left(\bar{\beta}, \beta^{*}, \alpha^{*}\right)$ is a $\mathbb{E}$-morphism from $E(M)$ to $\mathcal{O}$. But each component is an isomorphism, as we have proved. Thus it is a (5-isomorphism.

Consequently $E$ is representative and the main theorem is proved. Q.E.D.

REMARK. (i) Given $\mathcal{O} \equiv\left(M^{*}, \tau, V\right)$, let us denote by $P(\mathcal{O})$ the pullback of $\mathcal{O}$ in Cat $(R)$ (as described in 2.4). It can be shown that $P$ is a functor from $\mathbb{E}$ to $\mathfrak{M}_{R}$, and that it is in fact a quasi-inverse of $E$. From this it follows that $E$ is an equivalence; however, this method involves a more complicated sort of diagram chasing.

(ii) Given $\mathcal{O} \equiv\left(M^{*}, \tau, V\right)$, define $\operatorname{Rank} \mathcal{O}=\operatorname{Rank}_{R^{*}}\left(M^{*}\right)=\operatorname{dim}_{K}(V)$. Then our proof shows that $E$ is rank preserving; that is, for any $M \in \mathfrak{M}_{R}, \operatorname{Rank}_{R}(M)$ $=\operatorname{Rank}(E(M))$.

\section{Direct sums in $\mathfrak{E}$ and completely decomposable modules in $\mathfrak{M}_{R}$.}

5.1. Proposition. Let $\mathcal{O}_{i} \equiv\left(M_{i}^{*}, \tau_{i}, V_{i}\right)$ be a family of objects in $\mathbb{C}$ where $i$ runs through an index set $I$. Consider $\mathcal{O} \equiv\left(M^{*}, \tau, V\right)$ where $M^{*}=\bigoplus_{i \in I} M_{i}^{*}, \tau=\bigoplus_{i \in I} \tau_{i}$, and $V=\bigoplus_{i \in I} V_{i}$. Then $\mathcal{O} \in \mathrm{obj}(\mathbb{E})$ and $\mathcal{O}$ is the direct sum of the $\mathcal{O}_{i}$.

Proof. We have only to check the rationality and ampleness conditions for $\tau$. Clearly $\tau$ carries a suitable (and hence any $K$-linearly independent set of $V$ ) 
$K$-linearly independent set of $V$ into a $K^{*}$-linearly independent set, since it is the direct sum of maps having a similar property. Also

$$
\begin{aligned}
K^{*} \cdot \tau(V) & =K^{*}\left(\bigoplus_{i \in I} \tau_{i}\right)\left(\bigoplus_{i \in I} V_{i}\right)=K^{*} \bigoplus_{i \in I}\left(\tau_{i}\left(V_{i}\right)\right) \\
& =\bigoplus_{i \in I} K^{*} \tau_{i}\left(V_{i}\right)=\bigoplus_{i \in I} M_{i}^{*} \otimes K^{*}=M^{*} \otimes K^{*}
\end{aligned}
$$

Furthermore,

$$
\begin{aligned}
\tau\left(\bigoplus_{i \in I} V_{i}\right) \cap \lambda\left(\bigoplus_{i \in I} M_{i}^{*}\right) & =\left(\bigoplus_{i \in I}\left(\tau_{i}\left(V_{i}\right)\right) \cap\left(\bigoplus_{i \in I} \lambda_{i}\left(M_{i}^{*}\right)\right)\right. \\
& \supseteq \bigoplus_{i \in I}\left(\tau_{i}\left(V_{i}\right) \cap \lambda_{i}\left(M_{i}^{*}\right)\right),
\end{aligned}
$$

which implies both the ampleness conditions.

In order to show that $\mathcal{O}$ is a direct sum, we define maps $\hat{u}_{i}: \mathcal{O}_{i} \rightarrow \mathcal{O}$ for $i \in I$, where $\hat{u}_{i} \equiv\left(u_{i}: M_{i}^{*} \rightarrow M^{*}, u_{i} \otimes \mathrm{id}: M_{i}^{*} \otimes K^{*} \rightarrow M^{*} \otimes K^{*}\right.$, incl $\left._{i}: V_{i} \rightarrow V\right) ; u_{i}$ and incl $_{i}$ are the usual canonical injections.

It is easy to check that $\left(\mathcal{O}, \hat{u}_{i}\right)_{i \in I}$ is the direct sum of $\left(\mathcal{O}_{i}\right)_{i \in I}$.

5.2. Definition. $\mathcal{O} \equiv\left(M^{*}, \tau, V\right)$ is said to be completely decomposable whenever $\mathcal{O} \cong \oplus_{i \in I} \mathcal{O}_{i}$ where $\mathcal{O}_{i} \equiv\left(M_{i}^{*}, \tau_{i}, K\right)$ (hence $\left.\operatorname{Rank}\left(\mathcal{O}_{i}\right)=1\right)$.

5.3. Proposition. $M \in \mathfrak{M}_{R}$ is a completely decomposable module if and only if $E(M)$ is completely decomposable in $\Subset$.

Proof. $E$ is an equivalence by our main theorem.

5.4. Definition. Given $M \in \mathfrak{M}_{R}$ let $\lambda: M \rightarrow M \otimes K$ be the canonical monomorphism. A basis $\left\{e_{i}\right\}_{i \in I}$ of $M \otimes K$ is called a splitting basis for $M$ if there exists a complete decomposition $M=\bigoplus_{i \in I} A_{i}$ such that (i) $\lambda_{i}=\lambda \mid A_{i}: A_{i} \rightarrow K e_{i}$ for $i \in I$ and (ii) $\lambda=\oplus \lambda_{i}$.

REMARK. If $M$ is completely decomposable, a splitting basis always exists.

5.5. Proposition. $\mathcal{O} \equiv\left(M^{*}, \tau, V\right)$ in $\mathfrak{E}$ is completely decomposable if and only if there exists a basis $\left\{e_{i}\right\}_{i \in I}$ of $V$ such that $\left\{\tau\left(e_{i}\right)\right\}_{i \in I}$ is a splitting basis for $M^{*}$.

Proof. Suppose $\mathcal{O} \cong \bigoplus_{i \in I} \mathcal{O}_{i}$ and $\mathcal{O}_{i} \equiv\left(M_{i}^{*}, \tau_{i}, K\right)$. By definition $M^{*}=\bigoplus_{i \in I} M_{i}^{*}$, $\tau=\bigoplus_{i \in I} \tau_{i}$ and $\bigoplus_{i \in I} K=V$.

Conversely, suppose there exists a basis $\left\{e_{i}\right\}_{i \in I}$ of $V$ such that $\left\{\tau\left(e_{i}\right)\right\}_{i \in I}$ is a splitting basis for $M^{*}$. Then $\left\{\tau\left(e_{i}\right)\right\}_{i \in I}$ is a $K^{*}$-basis for $M^{*} \otimes K^{*}$, and $\tau=\oplus \tau_{\imath}$ where $\tau_{i}=\tau \mid K e_{i}$. Also im $\left(\tau_{i}\right) \subseteq K^{*} \tau\left(e_{i}\right)$. Let $M^{*}=\bigoplus_{i \in I} M_{i}^{*}$ be the complete decomposition of $M^{*}$ with respect to this splitting basis. Put $\mathcal{C}_{i} \equiv\left(M_{i}^{*}, \tau_{i}, K e_{i}\right)$. Now it is obvious that $\mathcal{O}=\bigoplus_{i \in I} \mathcal{O}_{i}$, proving that $\mathcal{O}$ is completely decomposable.

Any $K$-linear transformation $\tau: K \rightarrow K^{*}$ is actually the restriction of a $K^{*}$-linear transformation $\tau^{*}: K^{*} \rightarrow K^{*}$, which is just scalar multiplication. Hence by choosing the basis suitably (or "normalizing" the basis), we can assume $\tau_{i}=$ incl: $K \rightarrow K^{*} e_{i}$ in each of $\mathcal{O}_{i}$ in the proof of Proposition 5.5 above. 
Hence we have the following important corollary, which we record only for finite rank.

5.6. Corollary. An object $\mathcal{O} \equiv\left(M^{*}, \tau, V\right)$ of finite rank in $\mathbb{E}$ is completely decomposable if and only if there exists a basis $\left\{e_{i}\right\}_{i \in I}$ of $V$ such that $\left\{\tau\left(e_{i}\right)\right\}_{i \in I}$ is a splitting basis for $M^{*}$ and the corresponding matrix for $\tau$ is the identity matrix.

6. Applications. From here on, $R$ will be a valuation ring of $K$ and $R^{*}$ will be a valuation ring of a chosen maximal immediate extension $K^{*}$ of $K$ (see [4, Theorem 1.3]).

6.1. Proposition. Under the above hypotheses, let $\left(M^{*}, \tau, V\right)$ be any triple where $M^{*} \in \mathfrak{M}_{R^{*}}$ and $\tau: V \longmapsto M^{*} \otimes K^{*}$ is a monomorphism satisfying the rationality condition; then $K\left(\tau(V) \cap \lambda\left(M^{*}\right)\right)=\tau(V)$. This shows that one of the ampleness conditions on the objects of $\mathbb{E}$ is superfluous.

Proof. Consider the diagram in Proposition 2.4.

Suppose $z \in V$, so that $\tau(z) \in M^{*} \otimes K^{*}$. Now there is a nonzero element $a^{*} \in R^{*}$ and a $y \in M^{*}$ such that $a^{*} \tau(z)=\lambda(y)$, where $\lambda: M^{*} \rightarrow M^{*} \otimes K^{*}$ is the canonical monomorphism. If $a \in R$ is such that $v(a)=v^{*}\left(a^{*}\right) \geqq 0$, then $a a^{*-1} y \in M^{*}$ and $\lambda\left(a a^{*-1} y\right)=a a^{*-1} \lambda(y)=a a^{*-1} a^{*} \tau(z)=a \tau(z)$. We may therefore assume without loss of generality that there exists $0 \neq a \in R$ such that $a \tau(z)=\lambda(y)$. Then

$$
\tau(a z)=a \tau(z)=\lambda(y), \quad a z \in V, y \in M^{*} .
$$

Thus $\tau(a z) \in \tau(V) \cap \lambda\left(M^{*}\right)$ and so

$$
\tau(z)=a^{-1} z(a z) \in K \cdot\left(\tau(V) \cap \lambda\left(M^{*}\right)\right) .
$$

Hence we have $\tau(V) \subseteq K\left(\tau(V) \cap \lambda\left(M^{*}\right)\right)$. The other inclusion is clear. Q.E.D.

REMARK. Under some additional assumptions, it will be shown later that the other ampleness condition is likewise superfluous.

\section{Baer-Kulikov-Kaplansky theorem.}

7.1. TheOREM. Suppose $M \in \mathfrak{M}_{R}$ is completely decomposable and $N$ is a pure submodule of $M$ of finite rank. Then $N$ is a direct summand of $M$, and $N$ is completely decomposable.

Proof. Let $M=\oplus_{i \in I} A_{i}$, and let $N$ be the pure submodule generated, say, by $\left\{x_{1} \cdots x_{n}\right\}$ in $M$. Since each $x_{j}$ has only finitely many nonzero components in the above decomposition of $M$, it is contained in a direct summand of $M$ which is completely decomposable and of finite rank. $N$ itself is therefore contained in a completely decomposable module of finite rank. Consequently we may assume without loss of generality that $M$ is of finite rank. 
Assume first that $N=A$ is of rank one and consider the following diagram, with $M=\bigoplus_{i=1}^{i=n} A_{i}$.

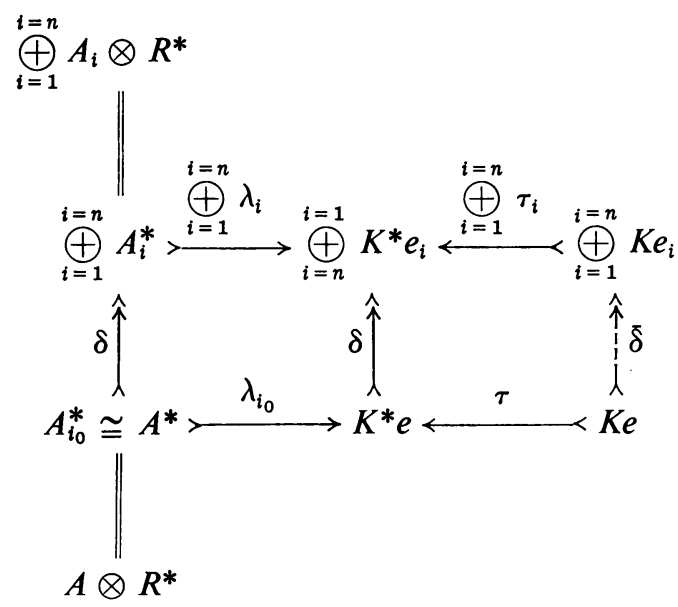

Here $\tau$ and all of the $\tau_{i}$ are natural inclusions by 5.6. Now $\bigoplus_{i=1}^{i=n} A_{i}^{*}$ is an $R^{*}$ module containing $A^{*}$ as a pure submodule. Hence $A^{*}$ is a direct summand of $\bigoplus_{i=n}^{i=1} A_{i}^{*}$ (by [5, Theorem 1.3, p. 338]). Thus $\bigoplus_{i=1}^{i=n} A_{i}^{*}$ is completely decomposable having $A^{*}$ as an indecomposable direct summand. It follows from Azumaya's theorem that $A^{*} \cong A_{i_{0}}^{*}$ for some index $i_{0}$ (see [2, Theorem 3.2]). Let $\delta: A^{*} \longrightarrow A_{i_{0}}^{*}$ be this isomorphism. From considerations of rank it follows that $\delta$ is actually a scalar multiplication by some $r \in K^{*}$ which is unique up to multiplication by units of $R^{*}$; we can therefore assume $r \in K$. Hence the right-hand end of the diagram can be completed so that the entire diagram commutes. Clearly $\left(A^{*}, \tau, K e\right)$ is a direct summand of $\left(\oplus_{i=1}^{n} A_{i}^{*}, \oplus \tau_{i}, \bigoplus_{i=1}^{n} K e_{i}\right)$. Hence $A$ is a direct summand of $\bigoplus_{i=1}^{n} A_{i}$.

Now let $N$ be any pure submodule of $M$ of rank $>1$, and assume the result is true for all pure submodules of $M$ having rank less than that of $N$. If $A$ is a pure submodule of rank one in $N$, then it is pure in $M$, hence a direct summand of $M$, and therefore a direct summand of $N$. So $N=A \oplus N_{1}, M=A \oplus M_{1}$, and $M_{1}=\bigoplus_{i=1}^{i=n} A_{i}, i \neq i_{0}$. Since $N_{1} \subseteq M_{1}$, by the induction hypothesis $N_{1}$ is completely decomposable and is a direct summand of $M$. Q.E.D.

Remark. The assumption that $N$ has finite rank cannot be dropped (see [5, Remark 3, p. 338]).

7.2. Corollary (First Baer-Kulikov-Kaplansky Theorem). If $M \in \mathfrak{M}_{R}$ is completely decomposable of countable rank and $N$ is a pure submodule of $M$, then $N$ is completely decomposable.

Proof. Let $N=\bigcup_{i=1}^{\infty} N_{i}$ where $N_{i}$ is a pure submodule of $N$ of rank $i$. Each $N_{i}$ is also pure in $N_{i+1}$. Furthermore, each $N_{i}$ is a direct summand of $M$ and is completely decomposable by Theorem 7.1. Hence $N_{i+1}=A_{i+1} \bigoplus_{j=1}^{n} A_{j}$ where $N_{i}$ 
$=\bigoplus_{j=1}^{n} A_{j}$. Thus by induction $N=\bigcup_{i=1}^{\infty} N_{i}=\bigoplus_{i=1}^{\infty} A_{i}$ and is completely decomposable. Q.E.D.

Kaplansky proved the following remarkable theorem [6, Theorem 1 and Remark on p. 373].

THEOREM. If $R$ is an integral domain and $M$ is a torsion free $R$-module which is a direct sum of modules of countable rank then any direct summand of $M$ is likewise a direct sum of modules of countable rank.

7.3. Corollary (BAer-Kulikov-Kaplansky Theorem). If $M \in \mathfrak{M}_{R}$ is completely decomposable, and $N$ is a direct summand of $M$, then $N$ is completely decomposable.

Proof. Using the previous theorem, $M$ can be assumed to have countable rank. But then the theorem follows from Corollary 7.2. Q.E.D.

8. A complete set of invariants. Given $\mathcal{O} \equiv\left(M^{*}, \tau, V\right)$ we have seen that one of the ampleness conditions on $\tau$ follows from the rationality of $\tau$. It is interesting to ask whether the condition of rationality implies the remaining ampleness condition. That this is not true in general is demonstrated by the following counterexample due to W. D. Geyer (oral communication).

EXAMPLE (GeYer). We define a valuation of rank two on the field $K=\boldsymbol{Q}(X)$ as follows: for any nonzero $f \in Q[X]$, there is a $g \in Q[X]$ such that $f=X^{m} g$ where $g(0)=2^{n} p / q \neq 0$ with $p$ and $q$ both odd; we define $v(f)=(m, n) \in Z \times Z$ and extend it to $Q(X)$ in the usual way.

It is easy to check that this is a valuation. In fact it corresponds to the following composite place on $Q(X)$

$$
Q(X) \underset{X=0}{\longrightarrow} Q \underset{2=0}{\longrightarrow} \frac{Z}{2} .
$$

Let the prime ideals in the valuation ring $R$ be denoted by $p, q$, respectively. Then we have $K \supset R \supset p \supset q \supset 0$. Let us take a maximal immediate extension $K^{*} \supset R^{*} \supset p^{*} \supset q^{*} \supset 0$. We note that $q \subset K$ and hence $Q_{2}$, the 2-adic completion with reference to the place $2=0$, can be assumed to be contained in $K^{*}$. Take $\alpha \in R^{*}$ such that $V^{*}(\alpha)=(0,0)$ with $\alpha \in Q_{2}, \alpha \notin Q$.

Consider the object $\mathcal{O} \equiv\left(M^{*}, \tau, V\right)$, where $M^{*}=K^{*} e_{1} \oplus q^{*} e_{2}, V=K e_{1} \oplus K e_{2}$ and $\tau: K e_{1} \oplus K e_{2} \rightarrow K^{*} e_{1} \oplus K^{*} e_{2}$ is given by the matrix $\tau \equiv\left(\begin{array}{cc}1 & 0 \\ \alpha & 1\end{array}\right)$.

Clearly $\tau$ satisfies the condition of rationality. However, it does not satisfy the ampleness condition, as we now prove. Let

$$
M=M^{*} \cap \tau(V)=\left\{(\lambda, \lambda \alpha+\mu): \lambda, \mu \in K, \lambda \alpha+\mu \in q^{*}\right\} .
$$

It is enough to show $R^{*} \cdot M \neq M^{*}$.

Now we claim that $\alpha-\delta \notin \boldsymbol{q}^{*}$ for any $\delta \in K$. If $\delta \in Q$, this is clear. If $\delta=X$, then by using the place $X=0$ we see that $\alpha-X \in q^{*}$ implies $\bar{\alpha}=\bar{X}=0$ in $\boldsymbol{Q}$; but $\alpha \notin \boldsymbol{Q}$ by choice, hence $\alpha-X \notin q^{*}$. By iteration, we conclude that $\alpha-\delta \notin q^{*}$ for any $\delta \in K$. 
Now $\lambda \alpha+\mu=q^{*} \in q^{*}$. We know $\alpha-\mu / \lambda=q^{*} / \lambda \notin q^{*}$ by the above argument. Hence $1 / \lambda \notin R_{q^{*}}$, so that $\lambda \in q^{*} \cap K=q$. Finally, from $\lambda \alpha+\mu \in q^{*}$ and $\alpha \lambda \in q^{*}$, we have $\mu \in \boldsymbol{q}^{*} \cap K=q$.

Consequently $\quad M=q f \oplus q e_{2} \quad$ where $f=e_{1}+e_{2}, \quad$ and $\quad R^{*} \cdot M=R^{*} q f \oplus R^{*} q e_{2}$ $=\boldsymbol{q}^{*} e_{1} \oplus \boldsymbol{q}^{*} e_{2} \varsubsetneqq K^{*} e_{1} \oplus \boldsymbol{q}^{*} e_{2}$.

Thus $\left(M^{*}, \tau, V\right)$ as given above satisfies the rationality condition, but not the ampleness condition.

Nevertheless by imposing some natural restrictions on the ring $R$, we can ensure that the ampleness condition will always follow from the rationality condition.

A valued field $(K, v)$ is called almost-maximal if every maximal immediate extension is a topological completion of $K$ with respect to the valuation. The corresponding valuation ring is then called an almost-maximal valuation ring.

Our present aim is to show that when $R$ is almost-maximal, rationality implies ampleness. Hereafter we will assume that $R$ is almost-maximal. We require some general results of a topological nature.

If $(K, v)$ be a valued field, then there is a uniformity defined locally at 0 by taking $V_{\gamma}(0)=\{x \mid x \in K: V(x)>\gamma\}$ for $\gamma \in \Gamma$, as a basis of neighborhoods at zero. In this way $K$ may be considered as a topological field.

Let $V$ be a $K$-space, and let $U$ be the family of all $R$-modules $M \subseteq V$ such that for each $x \in V$ there exists a nonzero $r \in R$ with $r x \in M$.

8.1. Proposition. $U$ defines a unique uniformity on $V$ so that $V$ becomes a Hausdorff topological vector space over $K$.

Proof. Clearly $\boldsymbol{U}$ defines a basis of neighborhoods at 0 . A set $S$ is then said to be open in $V$ if for each $x \in S$ there exists $M \in U$ such that $x+M \subseteq S$. It is obvious that these open sets constitute a topology on $V$.

Each $M$ is in particular an abelian group, so $M+M \subseteq M$ and addition is continuous.

We also note that if $M \in U$ and $0 \neq \alpha \in K$ then $\alpha M \in U$. Given $\alpha_{0} \in K$ and $x_{0} \in V$, we shall show for each $M \in U$, there exists $N \in U$ and $\gamma \in \Gamma$ such that $\alpha x \in \alpha_{0} x_{0}+M$, whenever $v\left(\alpha-\alpha_{0}\right)>\gamma$ and $x \in x_{0}+N$.

If $\alpha_{0}=0$, take $N=M$, and if $\alpha_{0} \neq 0$, take $N=M \cap M / \alpha_{0}$. In either case $N \in \boldsymbol{U}$ and there exists $0 \neq \beta \in R$ such that $\beta x_{0} \in N$. We can choose a $\gamma \in \Gamma$ such that $\gamma \geqq v(\beta)$. Suppose $v\left(\alpha-\alpha_{0}\right)>\gamma$ and $x-x_{0} \in N$; then $\left(\alpha-\alpha_{0}\right) x_{0}=\left(\left(\alpha-\alpha_{0}\right) / \beta\right) \beta x_{0} \in N$ and $\left(\alpha-\alpha_{0}\right)\left(x-x_{0}\right) \in N$. Moreover, $\alpha_{0}\left(x-x_{0}\right) \in \alpha_{0} N \cap M$. Consequently $\alpha x-\alpha_{0} x_{0}$ $=\left(\alpha-\alpha_{0}\right) x_{0}+\alpha_{0}\left(x-x_{0}\right)+\left(\alpha-\alpha_{0}\right)\left(x-x_{0}\right) \in M+M+M \subseteq M$.

Thus multiplication is also continuous.

Now $V$ is a topological vector space. To prove it is Hausdorff it is enough to prove it is $T_{1}$, that is, $\bigcap\{M: M \in U\}=(0)$. Let $\left(e_{i}\right)_{i \in I}$ be a basis of $V$ and consider $M_{0}=\bigoplus_{i \in I} R e_{i} \subseteq V$. Then clearly $\alpha M_{0} \in U$ for every $\alpha \in R$. Furthermore, there is no nonzero element of $M_{0}$ of infinite height; (i.e.) there is no nonzero $x \in M_{0}$ such that $x \in \alpha M_{0}$ for every $\alpha \in R$. 
Since $N=\bigcap_{\alpha \in R} \alpha M_{0} \subseteq M_{0}$ is the $R$-submodule of $M_{0}$ consisting of all elements of infinite height, it follows that $N=0$. Thus we have

$$
(0) \subseteq \bigcap\{M: M \in U\} \subseteq \bigcap_{\alpha \in R} \alpha M_{0}=(0) .
$$

8.2. Lemma. If $M \in U$ then $M$ is closed.

Proof. Let $\left(x_{\delta}\right)_{\delta \in \Delta}$ be a net in $M$ converging to $x \in V$. $M$ is a neighborhood of 0 , hence $x-x_{\delta} \in M$ eventually. But then $x=x-x_{\delta}+x_{\delta} \in M$. Q.E.D.

8.3. Proposition. Let $\left(K^{*}, v^{*}\right)$ be a maximal immediate extension of $(K, v)$ (since $(K, v)$ is almost-maximal, this is the ordinary completion) and let $V^{*}$ be a $K^{*}$-space. Suppose $V$ is a $K$-space in $V^{*}$ such that $K^{*} \cdot V=V^{*}$. Then $V$ is dense in $V^{*}$ (with respect to the $K^{*}$-uniformity on $V^{*}$ ).

Proof. Let $\bar{V}$ be the closure of $V$ in $V^{*}$. First observe that $\bar{V}$ is a $K$-space. Given $x \in \bar{V}$ and $\alpha \in K$, choose $x_{\delta} \in V$ such that $\left(x_{\delta}\right)_{\delta \in \Delta}$ converges to $x$. Since our spaces are Hausdorff, we can write this as $x=\lim x_{\delta}$ without ambiguity. Now $V^{*}$ may be considered as a $K$-space, so for every $\alpha \in K$ we have $\alpha x \in V^{*}$. But $\alpha x=\lim \left(\alpha x_{\delta}\right)$ by continuity, so $\alpha x \in \bar{V}$. By similar arguments we see that $\bar{V}$ is closed under addition. Secondly, $\bar{V}$ is also a $K^{*}$-space. Let $x \in \bar{V}, \alpha \in K^{*}$ and let $\left(\alpha_{\delta}\right)_{\delta \in \Delta}$ be a net in $K$ converging to $\alpha$. The $\left(\alpha_{\delta} \cdot x\right)_{\delta \in \Delta}$ is a net in $\bar{V}$ converging to $\alpha x \in V^{*}$; hence $\alpha x \in \bar{V}$.

Now $V \subseteq \bar{V}$ and $\bar{V}$ is a $K^{*}$-space, which implies $K^{*} \cdot V \subseteq \bar{V} \subseteq V^{*}$. Q.E.D.

8.4. ThEOREM. When $R$ is almost-maximal, rationality implies ampleness.

Proof. Let us identify all the images for simplicity and consider the following situation: $V^{*}$ is a $K^{*}$-space and $V$ is a $K$-space in $V^{*}$ such that $K^{*} \cdot V=V^{*}$. Now let $M^{*}$ be an $R^{*}$-module in $V^{*}$ such that $K^{*} \cdot M^{*}=V^{*}$. If $M=V \cap M^{*}$, we want to prove that $R^{*} \cdot M=M^{*}$.

Note that $M^{*}$ is a basic neighborhood of 0 in $V^{*}$, and so is $R^{*} \cdot M$ (the proof is similar to, and follows from, that of Proposition 6.1). Hence both $M^{*}$ and $R^{*} \cdot M$ are open. Given $x \in M^{*}$, there is a net $\left(x_{\delta}\right)_{\delta \in \Delta}$ in $V$ converging to $x$ by the previous proposition. Then $x-x_{\delta} \in M^{*}$ eventually. But $x \in M^{*}$, hence $x_{\delta} \in M^{*}$ eventually. Thus $x_{\delta} \in V \cap M^{*} \subseteq R^{*} \cdot\left(V \cap M^{*}\right)$ eventually, and so $x-x_{\delta} \in R^{*}\left(V \cap M^{*}\right)$ eventually.

Hence $x \in R^{*}\left(V \cap M^{*}\right)$. Q.E.D.

We devote the remainder of this section to the calculation of a complete set of invariants for the class of torsion-free modules over $R$ of at most countable rank. We continue to assume that $R$ is almost-maximal. To avoid further cumbersome notation, we still denote the corresponding categories by $\mathfrak{M}_{R}$ and $E\left(\mathfrak{M}_{R}\right)=\mathbb{C}$.

Let $\Gamma$ be the value group of $R$ (as well as that of $R^{*}$ ). Let upp $(\Gamma)=\{U \mid U \subseteq \Gamma: \gamma$ $\geqq \delta \in U$ implies $\gamma \in U\}$ be the set of upper classes of $\Gamma$. It is a monoid under the naturally induced operation of addition from $\Gamma$. There is a canonical imbedding of $\Gamma$ in upp ( $\Gamma)$ mapping $\gamma \in \Gamma$ into the upper class $(\gamma)=\{\lambda \mid \lambda \in \Gamma: \lambda \geqq \gamma\}$. If $U_{1}, U_{2} \in \operatorname{upp}(\Gamma)$ we write $U_{1} \sim U_{2}$ whenever there exists a $\gamma \in \Gamma$ such that $U_{1}+(\gamma)$ 
$=U_{2}$. This relation $\sim$ is an equivalence relation on upp $(\Gamma)$ which is compatible with addition. The quotient monoid $I(R)=$ upp $(\Gamma) / \sim$ is called the ideal class monoid of $R$.

Let $\mathfrak{N}$ be a complete set of representatives for the isomorphism classes of $R^{*}$ fractional ideals (that is, rank one $R^{*}$-modules of $K^{*}$ ). Then $(\mathfrak{N}, \otimes)$ is a monoid isomorphic to $I(R)$ (see [2, Theorem 2.2]). It is worth noting that $\mathfrak{A}$, being a set of $R^{*}$-fractional ideals, is linearly ordered by set inclusion.

Let $\mathfrak{M}_{\infty}$ be the following full subcategory of $\mathfrak{M}_{R^{*}}$. The objects of $\mathfrak{M}_{\infty}$ are $R^{*}$ modules of the form $\bigoplus_{i \in I} A_{i}$ with $A_{i} \in \mathfrak{A}$ and $|I| \leqq \aleph_{0}$, together with all the morphisms among them. By using the axiom of choice if necessary, we may assume the only isomorphisms in $\mathfrak{M}_{\infty}$ are automorphisms. Thus $\mathfrak{M}_{\infty}$ is a skeletal full subcategory of $\mathfrak{M}_{R^{*}}$. In fact we form all possible external direct sums out of $\mathfrak{A}$, with consideration only for the number and type of summands occurring in one such direct sum, to construct $\mathfrak{M}_{\infty}$.

Furthermore given any $M \in \mathfrak{M}_{R^{*}}$, let $M=\bigoplus_{j \in J} B_{j}$ be a complete decomposition of $M$ (see [5, Theorem 12] or [3, Proposition 7]). If $B_{j} \cong A_{i(j)}$ for every $j \in J$, where $A_{i(j)} \in \mathfrak{Q}$, let $M^{\prime}$ be the unique external direct sum formed from the family $\left\{A_{i(j)}\right\}$ such that $M^{\prime} \in \mathfrak{M}_{\infty}$. Now $M^{\prime} \cong M$ and $M^{\prime} \in \mathfrak{M}_{\infty}$. Hence $\mathfrak{M}_{\infty}$ is equivalent to $\mathfrak{M}_{R^{*}}$.

Hereafter $\left|\mathfrak{M}_{\infty}\right|$ will be used to denote the class (indeed the set) of modules in $\mathfrak{M}_{\infty}$.

Now given $M, N \in\left|\mathfrak{M}_{\infty}\right|$, we define $M+N=|M \oplus N|$ where $|M \oplus N|$ is the unique module in $\mathfrak{M}_{\infty}$ isomorphic to $M \oplus N$. Similarly we define $M \cdot N=|M \otimes N|$. Then $\left(\left|\mathfrak{M}_{\infty}\right|,+, \cdot\right)$ is a monoid-semiring.

Let $\boldsymbol{N}^{*}=\boldsymbol{N} \cup\{\infty\}$ be the usual abelian monoid of integers with infinity. We identify $\infty$ with $\aleph_{0}$. Let $N^{*}[I(R)]=\left\{s=\sum s_{\lambda} X^{\lambda} \mid s_{\lambda} \in N^{*}, \lambda \in I(R)\right\}$ be the set of all formal symbols of the indicated type with the usual definitions of componentwise equality and addition, and convolution multiplication; in other words, the monoid semiring on $I(R)$.

8.5. Proposition. $\left(\left|\mathfrak{M}_{\infty}\right|,+, \cdot\right) \cong\left(N^{*}[I(R)],+, \cdot\right)$.

Proof. As already remarked $(\mathfrak{A}, \otimes) \cong(I(R),+)$. Let $\boldsymbol{Y}=\left\{X^{\lambda} \mid \lambda \in I(R)\right\} \subseteq$ $N^{*}[I(R)]$; clearly $(I(R),+) \cong(Y, \cdot)$. Now $\left|\mathfrak{M}_{\infty}\right|$ is freely generated over $\mathfrak{A}$ and $N^{*}[I(R)]$ is freely generated over $Y$. Hence the induced extended map is clearly an isomorphism. Q.E.D.

REMARK. (i) Given $s \in N^{*}[I(R)]$, say $s=\sum s_{\lambda} X^{\lambda}$, define the trace of $s$ to be $\operatorname{Tr}(s)=\sum s_{\lambda} \in N^{*}$.

(ii) If $T y$ is the isomorphism established in Proposition 8.5, and $T y(M)=s$, then we say that $M \in\left|\mathfrak{M}_{\infty}\right|$ is of type s. In this case $\operatorname{Rank}(M)=\operatorname{Tr}(T y(M))$.

8.6. Definition. Let $\overline{\mathfrak{C}}$ be the full subcategory of $\mathbb{C}$ consisting of objects $\mathcal{O} \equiv\left(M^{*}, \tau, V\right) \in \mathbb{E}$ where

(i) $M^{*}=\bigoplus_{i \in I} A_{i} \in\left|\mathfrak{M}_{\infty}\right|$,

(ii) $M^{*} \otimes_{R^{*}} K^{*}=\bigoplus_{i \in I} K^{*} e_{i}$ with $A_{i} \subseteq K^{*} e_{i}$, and

(iii) $V=\bigoplus_{i \in I} K e_{i} \subseteq \bigoplus_{i \in I} K^{*} e_{i}$. 
8.7. Proposition. $\overline{\mathfrak{C}}$ is a representative subcategory of $\mathfrak{C}$, hence is equivalent to $\mathfrak{C}$.

Proof. Given $\mathcal{O} \equiv\left(M^{*}, \tau, V\right)$ in $\mathfrak{E}$. Consider the following diagram:

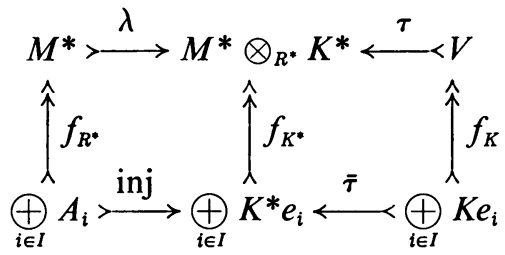

where $\oplus_{i \in I} A_{i}$ is the unique object in $\left|\mathfrak{M}_{\infty}\right|$ isomorphic to $M^{*}$ by the isomorphism $f_{R^{*}}$. Let $f_{K^{*}}: \bigoplus_{i \in I} K^{*} e_{i} \rightarrow M^{*} \bigotimes_{R^{*}} K^{*}$ be the unique $K^{*}$-isomorphism induced by $f_{R^{*}}$. Since $f_{R^{*}}$ is bijective, $f_{K^{*}}$ is also bijective and the first square commutes.

$f_{K^{*}}$ and $\tau$ can be considered as concurrent $K$-morphisms in Cat $(K)$. Let $\bar{V}$ be the pullback (unique up to isomorphism). Since Cat $(K)$ is an abelian category and $\tau$ is injective, it follows that $\bar{\tau}$ is injective. Similarly since $f_{K^{*}}$ is bijective, $f_{K}$ is also bijective. Hence $\bar{V} \cong V$. Thus $\bar{V}$ can be replaced by $\oplus_{i \in I} K e_{i}$ with suitable changes in the maps $\bar{\tau}$ and $f_{K}$, which may still be denoted by $\bar{\tau}$ and $f_{K}$ respectively, without causing any confusion.

$$
\begin{aligned}
f_{K^{*}}\left[K^{*} \cdot \bar{\tau}\left(\bigoplus_{i \in I} K e_{i}\right)\right] & =K^{*}\left[f_{K^{*}} \circ \bar{\tau}\left(\bigoplus_{i \in I} K e_{i}\right)\right] \\
& =K^{*} \cdot \tau \circ f_{K}\left(\bigoplus_{i \in I} K e_{i}\right)=K^{*} \cdot \tau(V) \\
& =M^{*} \bigotimes_{R^{*}} K^{*}=f_{K^{*}}\left(\bigoplus_{i \in I} K^{*} e_{i}\right) .
\end{aligned}
$$

But $f_{K^{*}}$ is a bijection, hence $K^{*} \cdot \bar{\tau}\left(\bigoplus_{i \in I} K e_{i}\right)=\bigoplus_{i \in I} K^{*} e_{i}$.

(ii) Now $\tau, f_{K}, f_{K^{*}}$ all preserve the linear independence of elements of the domain even under the extension of the scalars in the range. Hence by commutativity $\bar{\tau}$ must have the same property. So $\bar{\tau}$ carries $K$-linearly independent sets of elements into $K^{*}$-linearly independent sets in the range.

Thus $\bar{\tau}$ satisfies the rationality condition. But by assumption $R$ is almostmaximal. Hence $\tau$ also satisfies ampleness and $\overline{\mathcal{O}} \equiv\left(\oplus_{i \in I} A_{i}, \bar{\tau}, \bigoplus_{i \in I} K^{*} e_{i}\right) \in \operatorname{obj}(\overline{\mathcal{C}})$ is isomorphic to $\mathcal{O}$. Thus $\widetilde{\mathbb{E}}$ is a representative subcategory of $\mathfrak{E}$, hence it is equivalent to $\Subset$.

Combining this with Remark (ii) at the end of $\S 4$ and Theorem $\mathrm{A}$, we have

8.8. COROLlARY. $\mathfrak{M}_{R}$ and $\overline{\widetilde{C}}$ are equivalent categories.

REMARK. (i) It should be emphasized that $\overline{\mathcal{E}}$ is not canonical, since it depends on our initial choice of $\mathfrak{A}$.

Since $\mathfrak{M}_{\infty}$ is skeletal, it is clear that for each $\bigoplus_{i \in I} K^{*} e_{i}$, there exists one, and only one, $R^{*}$-submodule of $\oplus_{i \in I} K^{*} e_{i}$ belonging to $\left|\mathfrak{M}_{\infty}\right|$ of a given preassigned type $s$. Hence we can make the following definition:

8.9. Definition. Suppose $\left(M_{i j}\right) \in \mathrm{GL}\left(n, K^{*}\right), 0<n \leqq \infty$. Then $\left(m_{i j}\right)$ is of type $s$ whenever the associated linear transformation $T\left(m_{i j}\right): \bigoplus_{i=1}^{i=n} K^{*} e_{i} \rightarrow \bigoplus_{i=1}^{i=n} K^{*} e_{i}$ leaves the unique $R^{*}$-submodule of type $s$ in $\bigoplus_{i=1}^{i=n} K^{*} e_{i}$ invariant. 
Now given an object $\mathcal{O} \equiv\left(\bigoplus_{i=1}^{i=n} A_{i}, \tau, \bigoplus_{i=1}^{i=n} K e_{i}\right)$ in $\overline{\mathbb{E}}$, the basis of $\bigoplus_{i=1}^{i=n} K e_{i}$ (and of $\left.\bigoplus_{i=1}^{i=n} K^{*} e_{i}\right)$ is canonically fixed, so $\tau$ can be thought of as belonging to GL $\left(n, K^{*}\right)$.

Let $\mathrm{GL}(K)$ be the set of all invertible matrices over $K$ of at most countable rank, and let $\mathrm{GL}\left(K^{*}\right)$ be defined analogously.

Let $G \equiv\left\{\left(s,\left(m_{i j}\right)\right) \mid s \in N^{*}[I(R)],\left(m_{i j}\right) \in \operatorname{GL}\left(K^{*}\right)\right.$ and $\left.\operatorname{Tr}(s)=\operatorname{Rank}\left(m_{i j}\right)\right\}$. We define a binary relation on $G$ as follows: $\left(s_{1},\left(m_{i j}\right)\right) \sim\left(s_{2},\left(n_{l k}\right)\right)$ whenever

(a) $s_{1}=s_{2}$ and $\operatorname{Tr}\left(s_{1}\right)=\operatorname{Tr}\left(s_{2}\right)=n$ say, where $0<n \leqq \infty$,

(b) there exists a $\left(p_{l i}\right) \in \mathrm{GL}\left(K^{*}\right)$ of type $s$ and rank $n$ and a $\left(q_{k j}\right) \in \mathrm{GL}(K)$ of rank $n$ such that

$$
\left(p_{l i}\right) \cdot\left(m_{i j}\right)=\left(n_{l k}\right) \cdot\left(q_{k j}\right)
$$

It is easy to check that $\sim$ is indeed an equivalence relation. We put $N^{*}[I(R)] * \mathrm{GL}\left(K^{*}\right) \equiv G / \sim$. Let $\pi: G \rightarrow G / \sim$ be the natural projection.

Now we come to the final theorem of this section.

8.10 THEOREM. $N^{*}[I(R)] * \mathrm{GL}\left(K^{*}\right)$ is a complete system of invariants for $\mathfrak{M}_{R}$.

Proof. By Corollary 8.8 , it is enough to prove the theorem for $\overline{\mathfrak{C}}$. We define the correspondence as follows.

Given $\mathcal{O} \equiv\left(\oplus A_{i}, \tau, \oplus K e_{i}\right)$ we put $\phi(\mathcal{O})=\pi\left(T y\left(\oplus A_{i}\right), \tau\right) \in N^{*}[I(R)]_{*} \operatorname{GL}\left(K^{*}\right)$ where $\left(T y\left(\oplus A_{i}\right), \tau\right) \in G$.

Let $\mathcal{O}_{1} \equiv\left(\oplus A_{i}, \tau_{1}, \oplus K e_{i}\right) \cong \mathcal{O}_{2} \equiv\left(\oplus B_{j}, \tau_{2}, \oplus K e_{j}\right)$. Then they have the same rank and $\oplus A_{i} \cong \oplus B_{j}$ in $\left|\mathfrak{M}_{\infty}\right|$, so $\oplus A_{i}=\oplus B_{j}$. Now if $s=T y\left(\oplus A_{i}\right)=T y\left(\oplus B_{j}\right)$, then $f_{K^{*}} \mid \oplus A_{i}=f_{R^{*}}$ is an automorphism of $\oplus A_{i}$. Hence $f_{K^{*}}$ is of type $s$. Also $\tau_{2} \circ f_{K}=f_{K^{*}} \circ \tau_{1}$ is one of the basic relations for a $\left({ }^{2}\right.$-morphism. By means of the canonical basis this is precisely the matrix equality for $\tau_{1} \sim \tau_{2}$. Hence $\phi\left(\mathcal{O}_{1}\right)$ $=\pi\left(s, \tau_{1}\right)=\pi\left(s, \tau_{2}\right)=\phi\left(\mathcal{O}_{2}\right)$ in $N^{*}[I(R)] * \mathrm{GL}\left(K^{*}\right)$. Thus isomorphic objects have the same invariants.

Conversely, let $\mathcal{O}_{1}, \mathcal{O}_{2} \in \overline{\mathcal{C}}$ such that $\phi\left(\mathcal{O}_{1}\right)=\phi\left(\mathcal{O}_{2}\right)$. Then $\phi\left(\mathcal{O}_{1}\right)=\pi\left(T y\left(\oplus A_{i}\right), \tau_{1}\right)$ $=\pi\left(T y\left(\oplus B_{j}\right), \tau_{2}\right)=\phi\left(\mathcal{O}_{2}\right)$ so (i) $T y\left(\oplus A_{i}\right)=T y\left(\oplus B_{j}\right)=s$, say, and $n=\operatorname{Tr}(s)$, (ii) there exists $T_{1} \in \mathrm{GL}\left(n, K^{*}\right)$ of type $s$ and $T_{2} \in \mathrm{GL}(n, K)$ such that $T_{1} \circ \tau_{1}$ $=\tau_{2} \circ T_{2}$.

Then $T_{1}\left(\oplus A_{i}\right)=\oplus B_{j}$ and $\left(T_{1} \mid \oplus A_{i}, T_{1}, T_{2}\right)$ is the required isomorphism and $\mathcal{O}_{1} \cong \mathcal{O}_{2}$.

Now given $\left(s,\left(m_{i j}\right)\right)^{\sim} \in N^{*}[I(R)] * \mathrm{GL}\left(K^{*}\right)$, let $\bigoplus_{i=1}^{i=n} A_{i}$ be the unique $R$ submodule in $\bigoplus_{i=1}^{i=n} K^{*} e_{i}$ of type $s$ and consider $\mathcal{O} \equiv\left(\bigoplus_{i=1}^{i=n} A_{i},\left(m_{i j}\right), \bigoplus_{i=1}^{n} K e_{i}\right)$. Clearly $\mathcal{O} \in \overline{\mathbb{E}}$ and $\phi(\mathcal{O})=\left(s,\left(M_{i j}\right)\right)^{\sim}$. The theorem is therefore proved. Q.E.D.

9. A characterization of maximal valuation rings. Using homological methods, Matlis ([7], see also [8, p. 318, Theorem 2]) proved the following

THEOREM. A valuation ring $R$ is maximal if and only if every torsion-free $R$ module of rank two is completely decomposable.

We now give another proof without using homological methods.

If $R$ is maximal, then every torsion-free module of countable rank is completely decomposable (see [5, Theorem 12, p. 338] or [3, Proposition 7, p. 252]). 
Conversely if $R$ is not maximal, then $K^{*} \neq K$ and there exists $\alpha \in K^{*}-K$. Let $\left\{\alpha_{i}\right\}_{i \in I}$ be a pseudo-convergent sequence in $K$, which pseudo-converges to $\alpha$. Then $v^{*}\left(\alpha-\alpha_{i}\right)=v\left(m_{i}\right)$ (and $\left.m_{i} \in R\right)$ is a set of monotonically increasing set of elements in the value group. Let $\mathfrak{A}=\left\{x \in K: \exists i \in I, v(x) \geqq-v\left(m_{i}\right)\right\}$ and similarly we define $\mathfrak{A}^{*} \subseteq K^{*} . \mathfrak{A}$ is an $R$-module in $K$ and $\mathfrak{A}^{*}$ is an $R^{*}$-module in $K^{*}$. Further $\mathfrak{A} \otimes R^{*}$ $=\mathfrak{A} \cdot R^{*}=\mathfrak{A}^{*}($ see $[2$, Lemma 2.1$])$.

Consider the following diagram:

$$
\begin{array}{r}
V=K e_{1} \oplus K e_{2} \\
Y\left(\begin{array}{ll}
1 & 0 \\
\alpha & 1
\end{array}\right) \equiv \tau \\
M^{*}=R^{*} e_{1} \oplus \mathfrak{A} e_{2}^{*} \longrightarrow K^{*} e_{1} \oplus K^{*} e_{2}^{*}
\end{array}
$$

Since $\tau$ is invertible, it satisfies the rationality condition.

To prove the ampleness condition: Now $\tau(V)=\{(x, \alpha x+y) \mid$ for $x, y \in K\}$. Then $M=\tau(V) \cap\left(R^{*} \oplus \mathfrak{A}^{*}\right)=\left\{(x, \alpha x+y) \mid v(x) \geqq 0, v^{*}(\alpha x+y) \geqq-v\left(m_{i}\right)\right.$ for some $i$ and $x, y \in K\}$. For $x=1$ and $y=0$, we have

$$
(1,0) \in M, \text { hence } R^{*} \oplus(0) \subseteq R^{*} \cdot M \text {. }
$$

Also $\left(0,1 / m_{i}\right) \in M$ for every $i \in I$. Then given $x \in \mathfrak{A}^{*}$, there exists $i_{0}$ such that $x m_{i_{0}} \in R^{*}$ so that we have

$$
(0, x)=x \cdot m_{i_{0}}\left(0,1 / m_{i_{0}}\right) \in R^{*} \cdot M .
$$

Combining (1) and (2) we have $R^{*} \oplus \mathfrak{A}^{*}=R^{*} \cdot M$.

The other ampleness condition is always satisfied in view of Proposition 6.1 and so $\left(R^{*} \oplus \mathfrak{A}^{*}, \tau, K \oplus K\right)$ is an object in $\mathfrak{S}$ of rank two. Let $M$ be the pullback of the associated diagram.

Then we claim that $M$ is not decomposable. Suppose on the contrary $M \cong A \oplus B, A, B \subseteq K$. By 2.4(iii) and (iv) and 2.2, we have $M \otimes R^{*} \cong R^{*} \oplus \mathfrak{I}^{*}$. Also $(A \oplus B) \otimes R^{*} \cong A^{*} \oplus B^{*} \cong R^{*} \oplus \mathfrak{U}^{*}$. By Azumaya's theorem [2, Theorem 3.2] we have, say, $A^{*} \cong R^{*}$ and $B^{*} \cong \mathfrak{Y}^{*}$. Since $A$ and $B$ are of rank one, we have $A \cong R$ and $B \cong \mathfrak{A}$ (see [2, Theorem 2.2]) and consequently $M \cong R \oplus \mathfrak{A}$; let $f$ be this isomorphism. Since $E(M) \cong E(R \oplus \mathfrak{U}) \cong E(R) \oplus E(\mathfrak{U})$, we have the following diagram in Cat $(R)$.

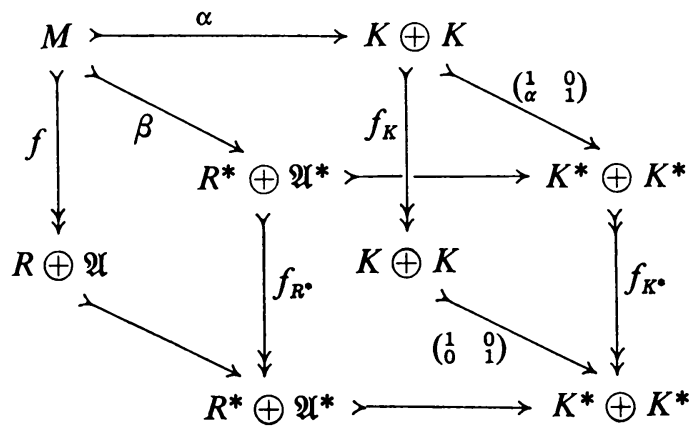


The unnamed arrows are natural injections.

Let

$$
f_{K^{*}} \equiv\left(\begin{array}{ll}
d_{1} & d_{2} \\
d_{3} & d_{4}
\end{array}\right) \quad \text { where } d_{i} \in K^{*}
$$

and

$$
f_{K} \equiv\left(\begin{array}{ll}
c_{1} & c_{2} \\
c_{3} & c_{4}
\end{array}\right) \quad \text { where } c_{i} \in K
$$

Now $f_{R^{*}}=f_{K^{*}} \mid R^{*} \oplus \mathfrak{A}^{*}$. Also we have

$$
\left(\begin{array}{l}
f_{K^{*}}\left(e_{1}^{*}\right) \\
f_{K^{*}}\left(e_{2}^{*}\right)
\end{array}\right)=\left(\begin{array}{ll}
d_{1} & d_{2} \\
d_{3} & d_{4}
\end{array}\right)\left(\begin{array}{l}
e_{1}^{*} \\
e_{2}^{*}
\end{array}\right)
$$

Hence $f_{K} *\left(e_{1}^{*}\right)=d_{1} e_{1}^{*}+d_{2} e_{2}^{*} \in R^{*} \oplus \mathfrak{A}^{*}$ with $d_{1} \in R^{*}, d_{2} \in \mathfrak{U}^{*}$. We claim $d_{1}$ is a unit: if not let $v^{*}\left(d_{1}\right) \geqq \varepsilon>0 . \varepsilon \in \Gamma$ and let $v^{*}\left(d_{2}\right)>v^{*}(d)$ with $d \in \mathfrak{A} *$-such a $d$ exists, since $\mathfrak{A}^{*}$ is clearly not principal. Without loss of generality assume $v^{*}\left(d_{2} / d\right)$ $\geqq \varepsilon$. Then $d_{1} e_{1}^{*}+d_{2} e_{2}^{*}=\eta\left(\left(d_{1} / \eta\right) e_{1}^{*}+\left(d_{2} / \eta\right) e_{2}^{*}\right)$ with $\eta \in R^{*}, v^{*}(\eta)=\varepsilon$ and $d_{2} / \eta \in \mathfrak{A}^{*}$; which contradicts the fact that $f_{K^{*}} \cdot\left(e_{1}^{*}\right)$ is not divisible by any element of $R$. Also $f_{K^{*}}\left(e_{2}^{*}\right)=d_{3} e_{1}^{*}+d_{4} e_{2}^{*}$ is divisible by $m_{i}$. Hence $d_{3} / m_{i} \in R^{*}$ for $i \in I$.

Now let us consider the inverse of $f_{R^{*}}$ which has the matrix

$$
\left(\begin{array}{cc}
d_{4} / \delta & -d_{2} / \delta \\
-d_{3} / \delta & d_{1} / \delta
\end{array}\right)
$$

where $\delta$ is the determinant. As before, we have $d_{4} / \delta$ is a unit and $d_{3} / \delta m_{i} \in R^{*}$ $\forall i \in I$. Hence we have $d_{3} / d_{4} m_{i} \in R^{*} \forall i \in I$.

Now from the preceding diagram we have

$$
f_{K} \equiv\left(\begin{array}{ll}
d_{1} & d_{2} \\
d_{3} & d_{4}
\end{array}\right)\left(\begin{array}{ll}
1 & 0 \\
\alpha & 1
\end{array}\right)
$$

is a matrix over $K$. Consequently, we have

$$
d_{1}+d_{2}=k_{1} \in K, \quad d_{3}+d_{4}=k_{2} \in K, \quad d_{2}, d_{4} \in K .
$$

Finally,

$$
\alpha+d_{3} / d_{4}=k \in K \text {. }
$$

Now $\alpha-k=d_{3} / d_{4}$ and since $d_{3} / d_{4} m_{i} \in R^{*} \forall i \in I$ we have $v^{*}(\alpha-k)>v\left(m_{i}\right), \forall i \in I$.

Hence $\alpha-k$ is in the breadth of the pseudo-convergent sequence we have chosen, which implies $k$ is a pseudo-limit, contrary to our assumption that $\left\{\alpha_{i}\right\}$ has no pseudo-limit in $K$. Q.E.D.

\section{REFERENCES}

1. N. Bourbaki, Algèbre commutative. Chap. 1, Actualités Sci. Indust., no. 1290, Hermann, Paris, 1961. MR 36 \#146.

2. S. M. Fakhruddin, The Grothendieck group of modules of finite rank over a valuation ring, J. Algebra 17 (1971), 25-33. 
3. I. Fleischer, Modules of finite rank over prufer rings, Ann. of Math. (2) 65 (1957), 250-254. MR 19, 9.

4. I. Kaplansky, Maximal fields with valuations, Duke Math. J. 9 (1942), 303-321. MR 3, 264.

5. - Modules over Dedekind rings and valuation rings, Trans. Amer. Math. Soc. 72 (1952), 327-340. MR 13, 719.

6. - Projective modules, Ann. of Math. (2) 68 (1958), 372-377. MR 20 \#6453.

7. E. Matlis, Some properties of Noetherian domains of dimension one, Canad. J. Math. 13 (1961), 569-586. MR 24 \#A1288.

8. - The decomposability of torsion free modules of finite rank, Trans. Amer. Math. Soc. 134 (1968), 315-324. MR 37 \#6317.

\author{
QUEENS UNIVERSITY, \\ Kingston, Ontario, Canada \\ UNIVERSITY OF HAWAII, \\ Honolulu, Hawail 96822
}

